\title{
Integrating thermodynamics and kinetics of urban systems for regional studies
}

\author{
R. M. Pulselli, F. Morandi \& E. Tiezzi \\ Ecodynamics Group - Department of Chemistry, \\ University of Siena, Italy
}

\begin{abstract}
The use of monitoring techniques and the construction of maps are used in an attempt to transfer the knowledge acquired through data collection and numeric calculations towards a concrete approach, finalized in the comprehension and management of real territorial systems. How can human activity be monitored? How are flows of energy and material conveyed within the territory? How much of the flows that feed human activities in a region come from outside of the local system and from the global market? How are they arranged in space and with what intensity? How do spatial patterns of activity develop over time, and what direction is the system evolving in? The response to this type of investigation could offer a key for the interpretation of various regional systems and to detect their thermodynamic and kinetic profile. For instance, the conformation and disposition of intensively active systems within a region can be studied through the observation of the dynamics that they are involved in and that they generate and are represented through spatial patterns on a cartographic basis.
\end{abstract}

Keywords: emergy evaluation, energy systems diagrams, geo-demograph, urban dynamics.

\section{Introduction}

Tools exist that are able to detect, with great precision, any fragment of our cities. We can identify the course of every street, the dimension of every building, the shape of every urban infrastructure. Researchers in urban studies can detect the physical form of a given region through many means and graphically represent it. In a few minutes, with the Internet, we can have a clear picture of any place in the world from a satellite shot. 
If we wanted to draw a parallel between regional sciences and medical sciences, we could say that nowadays we know the anatomy of our cities, and we have the tools to observe, modify, and design them. However, we know little of the processes that develop within urban systems that, in many respects, resemble the metabolism of a living organism. To date, we do not have diagnostic tools that are able to observe all the dynamic phenomena generated by a community that operates in a region, and moves, feeds, uses resources and communicates $[1]$.

The movements of the population, transfer of goods, flows of energy and materials, and the variations in intensity of all these phenomena, linked to the progress of the activities of a society, are real processes that are difficult to observe and monitor. The perception of a persistent fluidity of urban systems has caused many researchers to develop a new study approach in order to understand the dynamic evolutionary properties of urban systems and to reveal their whole functioning [2].

\section{Investigating thermodynamics and kinetics of urban systems}

In this paper, we deal with aims and techniques for investigating dynamic processes that take place in a metropolitan system, trying to highlight the different characteristics of openness and complexity that may be found in the various areas. In particular, we have focused on two aspects that we have generically brought back to two parts of dynamic physics, thermodynamics and kinetics.

Thermodynamics is the science that studies the basic principles that regulate the transformation of energy and all the general mechanisms at the basis of life. In the case of a regional system, that is an open system, energy and material exchanges with the environment and external systems are the object of the investigation. In particular, previous studies based on environmental accounting methods were developed for representing the intensity of the use of environmental resources within a region on the basis of the inventory of the flows of energy and materials that feed the anthropic activities (a case study will be briefly discussed in paragraph 3 ). This kind of processing has allowed for the detection of a whole configuration that tells how cities and other parts of a territorial system absorb energy in order to feed themselves and keep in life, like Prigogine's dissipative structures [2, 3].

Kinetics is the science that studies movement or, in general, the circulation of mass. Kinetic theory was born to explain the essential characteristics of matter, based on the hypothesis that it is made up of countless small particles in movement. In the common application to gases, this theory allows us to calculate the macroscopic behavior of gas based on the microscopic properties (velocity, kinetic energy, etc.) of the molecules it is composed of. Chemical kinetics studies the variations of the concentration of reagents and products in time and can be established experimentally by observing, with appropriate tools, processes and chemical reactions. In this paper, an in-depth study of the variations of 
population density over time was the pretext for investigating the complexity of behaviors of the community that operates in an urban system and observing the strong correlations among the various parts of the system, among the population and the structures of the city. A whole configuration, obtained through the elaboration of statistical data gathered through the technology of mobile telecommunications, has been interpreted as a tangible expression of the general organization of an urban social system and of the level of dynamism that this sustains (paragraph 4).

Generally, it is verified that high and low intensities in the use of resources correspond, in the various areas and settlements, to high and low levels of dynamism and organization.

\section{Thermodynamics: urban systems in the macroscope}

Energy systems models by Howard Odum [4] are born with the aim of having great potential for application. The activities of a community and the heterogeneous whole of the processes of transformation within a region are supported by resources, in the ways in which first nature, and then the market, make them available. Odum called these entering flows "driving energies". In particular, the inventory of all the driving energies that sustain and determine the development of urban systems derive from an accurate observation of all the dynamics and main processes that take place in it. A representation in a diagram provides a synthetic description of the flows of resources and of the processes of transformation that occur throughout a territory [5,6]. Specifically, an energy

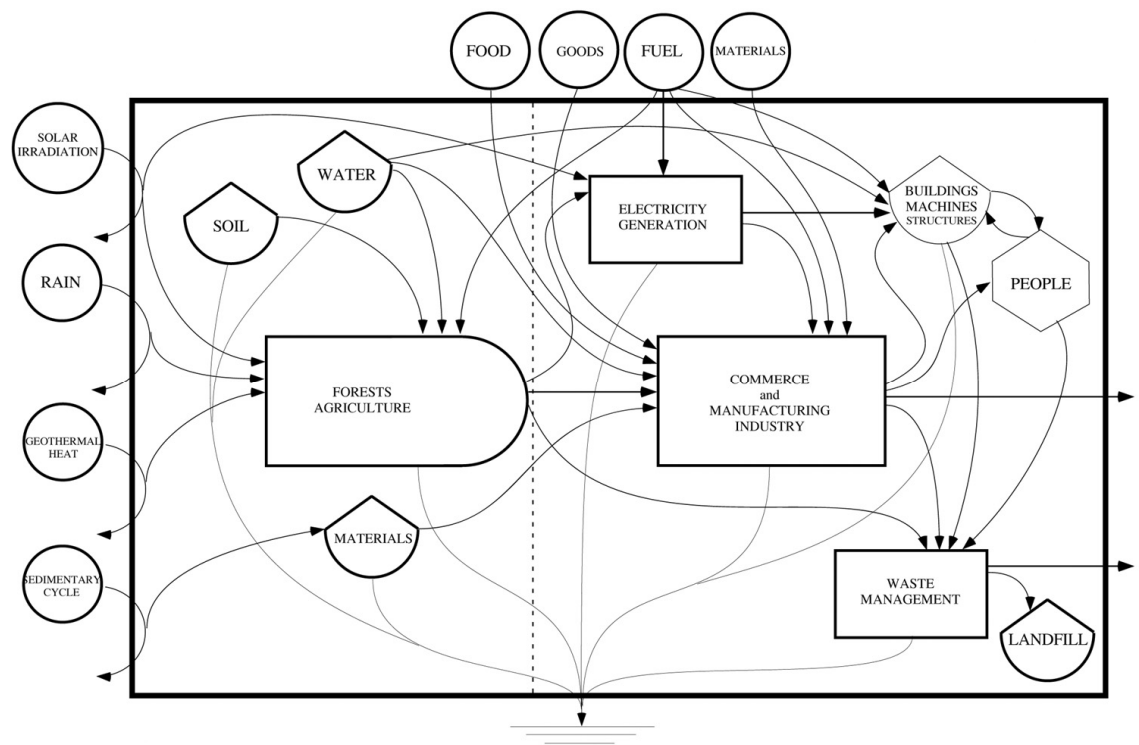

Figure 1: Energy system diagram of a region. 
system diagram provides a whole vision of the dynamics of a wide urban system that gathers diverse aspects and different sectors of activity in a single glance [2]. One of the most important innovations of this macroscopic vision of a territorial system is the evidence of a strong dependence of anthropic activities (right side of the diagram) on resources that directly derive from the environment. The thermodynamics of dissipative structures describes the general functioning of human dominated systems, interpreting them as elements included within the cycles of nature. The formation and the subsistence of structures, of the whole of services and urban functions, are processes that absorb resources from the external environment (represented by the arrows from different sources) and dissipate energy (represented by the arrows down to the heat sink).

Previous studies [5-7] were developed for measuring the environmental load of human activities on a territory, starting with the estimate of the flows of energy and matter necessary to sustain the levels of activity and well-being of the local community and to feed those productive, cultural, and social processes that represent the richness of a settled region and population. This information was

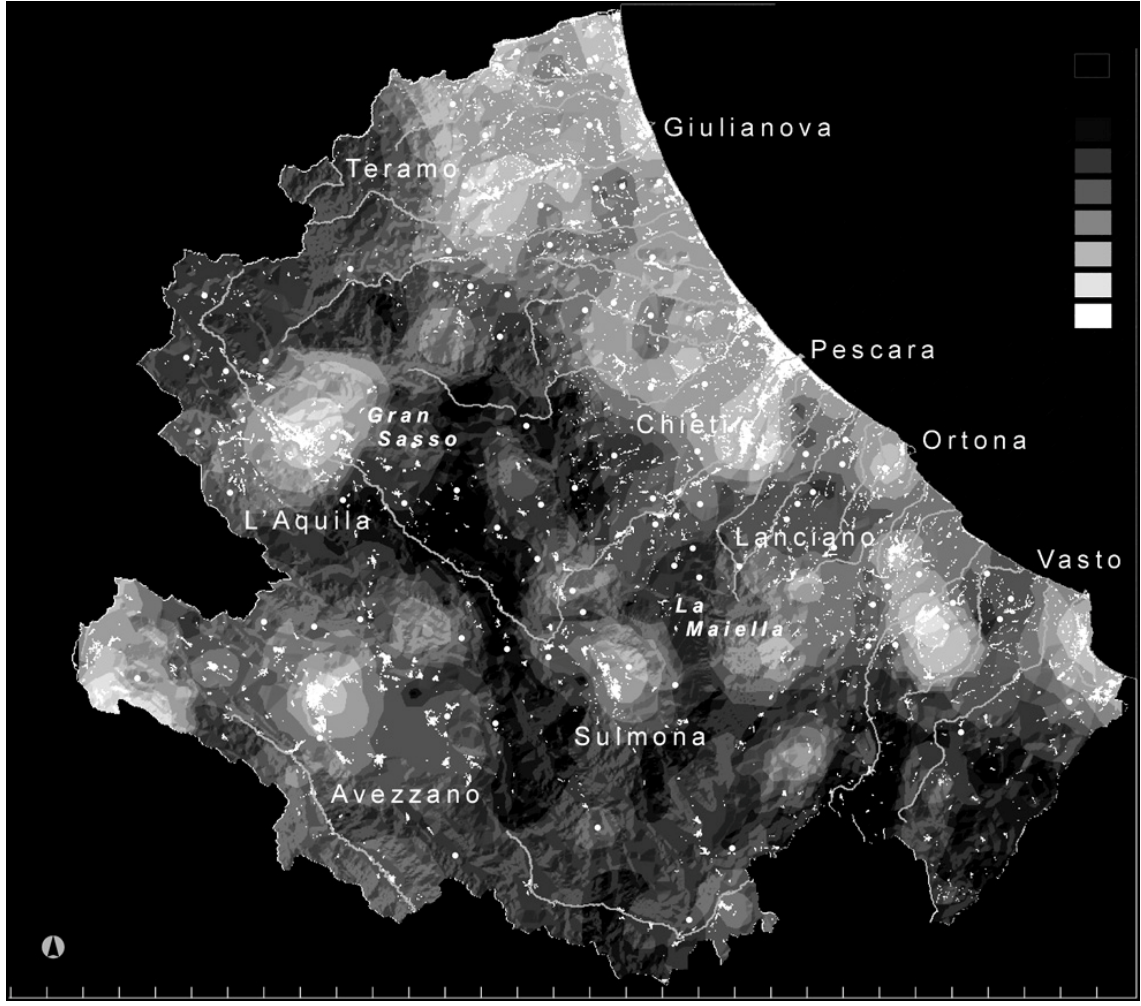

Figure 2: Emergy based map of the region Abruzzo. Highlighted areas indicate a high level of environmental resource use. 
related to the territory in order to give a more complete interpretation of space, and the use it is intended for, beyond the shape and geometry of the settlements. Through this type of investigation, it is possible: to know where and with what intensity the withdrawal and use of the necessary resources that sustain the system occur; to go more deeply into the effects of the activities that take place in each specific territorial area, and to know the ways resources are used, concretely observing their disposition throughout the territory; finally, to identify the most efficient or inefficient systems and processes [1].

A specific study has been created in this direction, developed on behalf of the Parks, Territory, Environment, Energy Management Sector in the Region of Abruzzo [7]. The analysis started with the inventory of the inputs that feed the territorial system, then, a thermodynamics based method of environmental accounting, namely Emergy Evaluation [4,8] was applied. An Emergy Evaluation - emergy stands for memory of energy - transforms units of energy and mass into units of equivalent solar energy (sej). By definition, the emergy $E m_{k}$ of an output flow $k$ of a process is:

$$
E m_{k}=\sum_{i=1}^{n} E n_{i} \times T r_{i}
$$

where $E n_{i}$ is the effective energy content of the $i$ th input and $\operatorname{Tr}_{\mathrm{i}}$ is the emergy per unit (transformity) of the $i$ th input. This is given in $\mathrm{sej} / \mathrm{J}$; otherwise, if the input is given in unit mass, its specific emergy content could be provided in $\mathrm{sej} / \mathrm{g}$.

This study had the aim of opening a new perspective on urban systems based on the observation of the relations that the various areas establish, as open systems, with the sources of resources they feed through. The goal is that of revealing a real but hidden aspect of a vast region that goes beyond traditional physical geography and, in a certain sense, mirrors its thermodynamic character.

As a result, this overall vision of the territory expresses the differences among the various ways of use of the land in terms of energies and resources involved, but also highlights the level of dependence on the various territorial sub-areas by a more or less intense activity of exchanges with other systems.

The intensity of flows of energy and matter, coming from the global market for the most part (approximately 70\% in the case of the Abruzzo Region), allows for the interpretation of each territorial sub-area as part of a system involved in multiple networks of processes that exceed the local dimension.

Table 1: $\quad$ Results from the emergy analysis of the region Abruzzo. Unit: solar emergy joule (sej).

\begin{tabular}{|c|c|c|c|c|c|}
\hline EMERGY & $\mathrm{R}$ & $\mathrm{N}$ & $\mathrm{L}=\mathrm{R}+\mathrm{N}$ & $\mathrm{F}$ & TOT \\
\hline REGION & $1,41 \times 10^{21}$ sej & $2,18 \times 10^{22}$ sej & $2,32 \times 10^{22}$ sej & $5,20 \times 10^{22} \mathrm{sej}$ & $7,52 \times 10^{22} \mathrm{sej}$ \\
\hline & $2 \%$ & $29 \%$ & $31 \%$ & $69 \%$ & $100 \%$ \\
\hline
\end{tabular}




\section{Kinetics: urban systems dynamics monitoring}

A research project, tested for the first time at the Massachusetts Institute of Technology in 2004 [9] and elaborated further by the researchers in Siena [1,2,10-12], experiments with an innovative technology for monitoring the variations of population density in real time, and observes its movements and dynamics in relation to the physical space of the city. In particular, within this study, the researchers intend to develop tools that will allow for the understanding of not only how the city users utilize urban space, but most importantly with what frequency and intensity. Instead of asking "how" the city space is utilized, this study aims to respond to the questions: "when", "for how long", and "how intensely".

The results of the research, reported in sequences of maps, generate a sort of "mobile geography" that reveals a shape of the organization of the territorial system studied and its evolution over time. The variations in intensity of activity can show, in a sequence, how a particular event, a localized disturbance, like a perturbation in a complex system, can provoke effects that are widespread, conditioning the functioning of a part of the system or the overall structure.

The study project we are presenting here was developed in 2007 with a contribution from Telecom Italia Mobile, which provided the data of the activity of the antennas present in the area under study, and was funded by the Department of Protection of Quality of Life and Environment of the Province of Pescara $[1,11,12]$. The subject of the research is a wide urbanized area that we identify as the metropolitan system of Pescara. Specifically, the area monitored
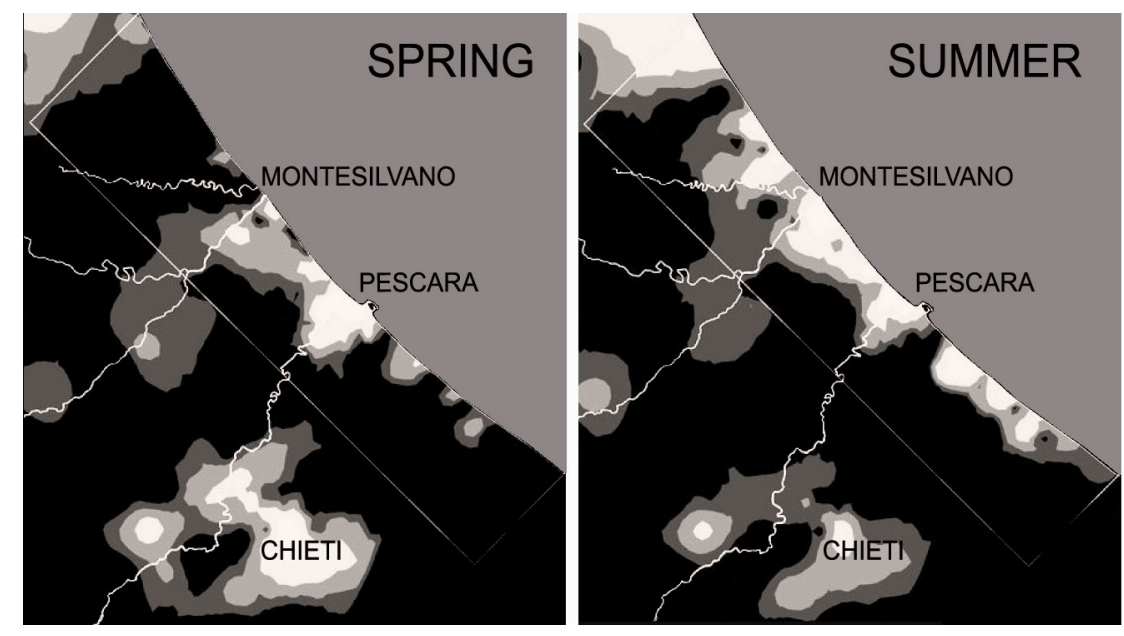

Figure 3: Geo-demographic patterns of the metropolitan area Pescara-Chieti in the region Abruzzo. Highlighted areas indicate a high level of activity. 
corresponds to approximately $860 \mathrm{Km}^{2}$ and includes, beyond the centers of Pescara and Chieti, the entire Province of Pescara and a $38 \mathrm{~km}$ coastal stretch.

The variety of times and spaces of use identify the characteristics of various areas, starting with the signaling of more or less active areas over the entire 24hour period or in specific time slots, with reference to workdays and holidays of the different seasons. Different results have emerged from the observation of the geo-demographic conformation of the metropolitan system of Pescara and of its evolution over time, in particular from the comparison of a workday in spring and a workday in mid-August, with their regularities and anomalies.

The geo-demographic configuration, identified through a synthetic interpretation of the dynamic maps, appears stable for a good part of the year. This organization is significantly modified in the summertime, revealing a completely different way of using the territory. In this period, the maps show a remarkable increase in activity along the coast, continuously involving a thin coastal strip, almost as if a single urban organism had been detected. These phenomena can be brought back to the well-known tourist-accommodation vocation of the Adriatic coast which, in the summer months, contributes to radically moving the center of the configuration revealed during the other seasons towards beach zones, imposing a new pattern of functioning on the territorial system.

The analysis of the overall data of the 24-hour period has allowed for the reconstruction of the percentage distribution of the activities in the different territorial areas, and therefore of the production, in addition to a qualitative description, of a quantitative estimate of the geo-demographic organization of the territory. Three greater areas have been identified, where $70-80 \%$ of the overall activity is concentrated; these areas correspond to those of the coast, and the axes along the valleys of Pescara and Tavo Rivers.

Further examination of the data has shown that, on a workday in spring, $50 \%$ of the activity of the entire territorial system was concentrated within a coastal strip. This percentage grows up to $65 \%$ in the summer period. In this strip of land, in fact, 936,960 contacts pass on one day in spring, compared to $1,146,592$ contacts on a summer day, with an increase of $22 \%$. The data increases in value if we consider that this area represents $20 \%$ of the entire area under examination.

\section{Conclusion}

On the whole, a significant correspondence exists between the level of organization, dynamism, and complexity of a system (meaning its kinetic aspects) and the level of use of environmental resources and of enthropic dissipation (thermodynamic aspects). Even in the case of a region, the more a system is organized, articulated, dynamic (high levels of population density), the greater will be the demand for the energy and matter (high level of emergy intensity) necessary to support the entirety of the activity that is executed within it.

On one hand, it is clear that urban systems correspond to high levels of complexity, organization and dynamism; cities are the place of cooperation, 
social well-being, the generation of ideas, the formation of culture, scientific and technological innovation and the economy of scale. On the other hand, one can observe that these systems, in order to survive, intensely utilize environmental resources, absorbing primary flows of energy and matter, and rid themselves of the entropy produced by the internal transformation processes in the form of heat, waste, refluent water, atmospheric emissions, and other waste products.

Addressing the research towards new investigative tools that study the various aspects linked to the dynamics of the contemporary city, beyond the physical shape of the settlement, is an important operation in order to try to open new pathways, oriented towards a deeper knowledge and management of urban systems that is more aware and sustainable. To this object is oriented the MoTo project, selected from the competition for research and innovation in the territorial and environmental field, and funded by the General Directorate of Territorial and Environmental Policies of the Tuscan Region [13].

\section{References}

[1] Pulselli R M, Romano P. Dinamiche dei Sistemi Urbani. Indagine di un'area metropolitana. Urban Systems Dynamics. Investigating a Metropolitan Area. Alinea, Firenze 2009.

[2] Pulselli R M, Tiezzi E. City Out of Chaos. Urban Self-organization and Sustainability. WIT Press, Southampton UK 2009. (Foreword by Paolo Portoghesi).

[3] I. Prigogine \& I. Stengers. The New Alliance. Galliamrs, Paris, 1979.

[4] H.T. Odum. Environment, Power and Society. Wiley, New York 1971.

[5] Pulselli R M, Pulselli F M, Rustici M. The emergy accounting of the Province of Siena: towards a thermodynamic geography for regional studies. Journal of Environmental Management 862008 342-353

[6] Pulselli R M, Rustici M, Marchettini N. An integrated holistic framework for regional studies: emergy based spatial analysis of the province of Cagliari. Environmental Monitoring and Assessment 1332007 1-13.

[7] Pulselli R M, Romano P, Bogunovich D, Pulselli F M. Integrating human and natural systems sustainably: emergy evaluation and visualization of the Abruzzo region. (Eds.) Brebbia C.A., Tiezzi E. Ecosystems and Sustainable Development VII. WIT Press. Southampton, UK 2009 273-278.

[8] H.T. Odum. Environmental Accounting: Emergy and Environmental Decision Making. Wiley, New York 1996.

[9] C. Ratti, R.M. Pulselli, S. Williams, D. Frenchman. Mobile Landscapes: using location data from cell phones for urban analysis. Environment and Planning B: Planning and Design 33(5) 727-748 2006.

[10] Pulselli R M, Ratti C, Tiezzi E. City out of chaos: social patterns and organization in urban systems. International Journal of Ecodynamics 1(2) 2006 125-134.

[11] Bastianoni S, Pulselli R M, Romano P, Pulselli F M. Dynamics and evolution of urban patterns: the evidence of the Mobile Landscapes project. 
(Ed.) Brebbia C A. Design \& Nature IV-Comparing Design in Nature with Science and Engineering. WIT Press. Southampton, UK 2008 253-260.

[12] Pulselli R M, Romano P, Ratti C, Tiezzi E. Computing urban mobile landscapes through monitoring population density based on cell-phone chatting. International Journal of Design \& Nature and Ecodynamics 3(2) 2008 121-134.

[13] Marchettini N, Romano P, Tiezzi E B P. An innovative survey of urban systems dynamics: the evidence of the MoTo project. (Ed.) Brebbia C A, Hernandez S, Tiezzi E. The Sustainable City VI. WIT Press. Southampton, UK 2010 (this issue). 